\title{
Preparation of the Nano Crystalline Powder from Iraqi Sand using the Sol-gel Technology
}

\author{
Adnan Meship Mahdi \\ Ministry of Education \\ Wasit, Iraq \\ Email: ad07802141877 [AT] gmail.com
}

\begin{abstract}
In this search nano silica powder was prepared by the bond fracturing of silica - oxygen using wet chemical method at PH 1-2 and by eliminate all undesired oxides by using ultrasound bath, and separating the compound by centrifuge, and washing the product with distilled water, and by separating and calcining the product at different temperatures it Weill is trail product with particle size (40-50) $\mathrm{nm}$, then XRD and AFM was to evaluate the product.
\end{abstract}

Keywords--- Nano Silica, ultrasonic, Scherer equation, Iraqi sand

\section{INTRODUCTION}

In this work the nano silica was synthesized by using sodium silicate $\mathrm{Na}_{2} \mathrm{SiO}_{3}\left(\mathrm{C}_{2} \mathrm{H}_{5}\right)_{4}$, hydrofluoric acid ,stiric acid by precipitation under controlled conditions using ethanol [1]. The properties of $\alpha+\beta$ nano silica and ether phases based are strongly dependent on the micro structure [2]. These substance can be thermo chemical processed to obtain a variety of microstructures depending on the amount of deformation, heat treatment temperature and rate of cooling ,mix imputation and the solution of $\mathrm{PH}[3]$. Iraqi Sand (SI) was treated acid nitric, fluoric acid to extract $\mathrm{SiO}_{2}$ [4].Nano Silica was produced by filtration and surface modification of this $\mathrm{SiO}_{2}[5]$. Nano Silica can be used as high-value added material for construction and medical service [6-8]. In recent years, considerable attention has been given to methods in which organic and inorganic catalytic surfaces are chemically modified in order to increase their usefulness in catalytic processes. The materials resulting from such chemical modifications have considerable potential as alternatives to conventional homogeneous and heterogeneous catalysts. Immobilization of transition metal complexes and metal clusters both provide reliable routes to a type of heterogeneous catalyst whose nature and mechanism may be more readily understood and potentially offer ways to manipulate metal particle and crystallite size to achieve new types of catalytic reactions [9] ( SI) as an inert material with a large surface area where the metal or its oxide is chemically incorporated and dispersed throughout matrix

\section{EXPERIMENTAL}

Provide and material

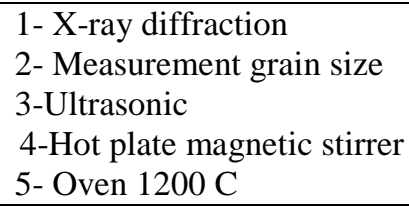

\author{
6- AFM ( Atomic Force Molecular ) \\ 7- Iraqi Sand (S I) \\ 8- Hydrofluoric Acid \\ 9-Stiric Acid \\ 10- Ethanol
}

The first washed thoroughly with water to remove the adhering s oil and dust. Edit 10 gm sample of SI was stirred in 0.1 $0.2 \mathrm{ml}$ of HF $42 \%$ for 144 hour \& $200 \mathrm{ml}$ Distilled water at room temperature. To orphan dissolve $0.5 \mathrm{gm}$ for stiric acid by $50 \mathrm{ml}$ ethanol bin to Ultrasonic for 20 min Mixing all this in reaction, attachment condenser to dominant the amount of deformation ,heat treatment temperature and rate of cooling and directly stirrer. That following observe append it looked explode in this reaction and checked the temperature at $60 \mathrm{c}$. The solution was filtered to remove undissolved particles , any undesirable material and washed by warm water another one .that following to dry up at (70 - 100) for (4-5) hour .It was then dried in an oven at $850^{\circ} \mathrm{C}$ for 1 hours. The prepared samples were characterized by X-Ray Diffractometry. A shape factor is used in $\mathrm{x}$-ray diffraction and crystallography to correlate the size of sub-micrometer particles, or crystallites, in a solid to the broadening of a peak in a diffraction pattern. In the Scherrer equation where $K$ is the shape 
factor, $\lambda$ is the $\mathrm{x}$-ray wavelength, $\beta$ is the line broadening at half the maximum intensity (FWHM) in radians, and $\theta$ is the Bragg angle; $[15,16] \tau$ is the mean size of the ordered (crystalline) domains, which may be smaller or equal to the grain size. The dimensionless shape factor has a typical value of about 0.9 , but varies with the actual shape of the crystallite. The Scherrer equation is limited to nano-scale particles. It is not applicable to grains larger than about $0.1 \mu \mathrm{m}$, which precludes those observed in most metallographic and ceramographic microstructures. It is important to realize that the Scherrer formula provides a lower bound on the particle size.

$\mathrm{G} . \mathrm{S}=\mathrm{K} \kappa / \beta \operatorname{Cos} \theta$

$\mathrm{K}=$ Constant $(0.9)$

$\kappa=1.5406 \mathrm{~A}^{\circ}$

$\beta=\mathrm{FWHM}$

$\Theta=$ Bragg angle

These properties were obtained from X-ray examination

ID: $40-1045$

Color White / $\mathrm{SiO}_{2}$ (101)

Quartz : syn

SYS : Hexagonal

[52.47 nm ] (101)

\section{RESULTS AND DISCUSSION}

SI obtained from the burning of the sand yielded white silica powder. The silica powder obtained is almost similar to that of the commercial silica gel in terms of the functional groups, the amorphous nature and also the physical structure [10]. Generally, SI silica contains metal oxides [11]. Treatment with concentrated HNO helps to reduce the impurities and leach out the metal oxides by forming nitrates which are easily dissolved in water. The acid treated sand, denoted as SI, dissolves in sodium hydroxide producing sodium silicate solution, as shown in Eq. (1), where $x$ is a variable that gives the ratio of the $\mathrm{SiO}_{2}$ to $\mathrm{Na}_{2} \mathrm{O}$ in the sodium silicate solution that affects its properties [12]. $\mathrm{SiO}_{2}$ $+2 \mathrm{NaOH} \rightarrow \mathrm{Na}_{2} \mathrm{SiO}_{3}+\mathrm{H}_{2} \mathrm{O}$

The sodium silicate solution was neutralized using nitric acid containing the metal to produce silica gel with the metal ion chemically incorporated into the silica matrix.
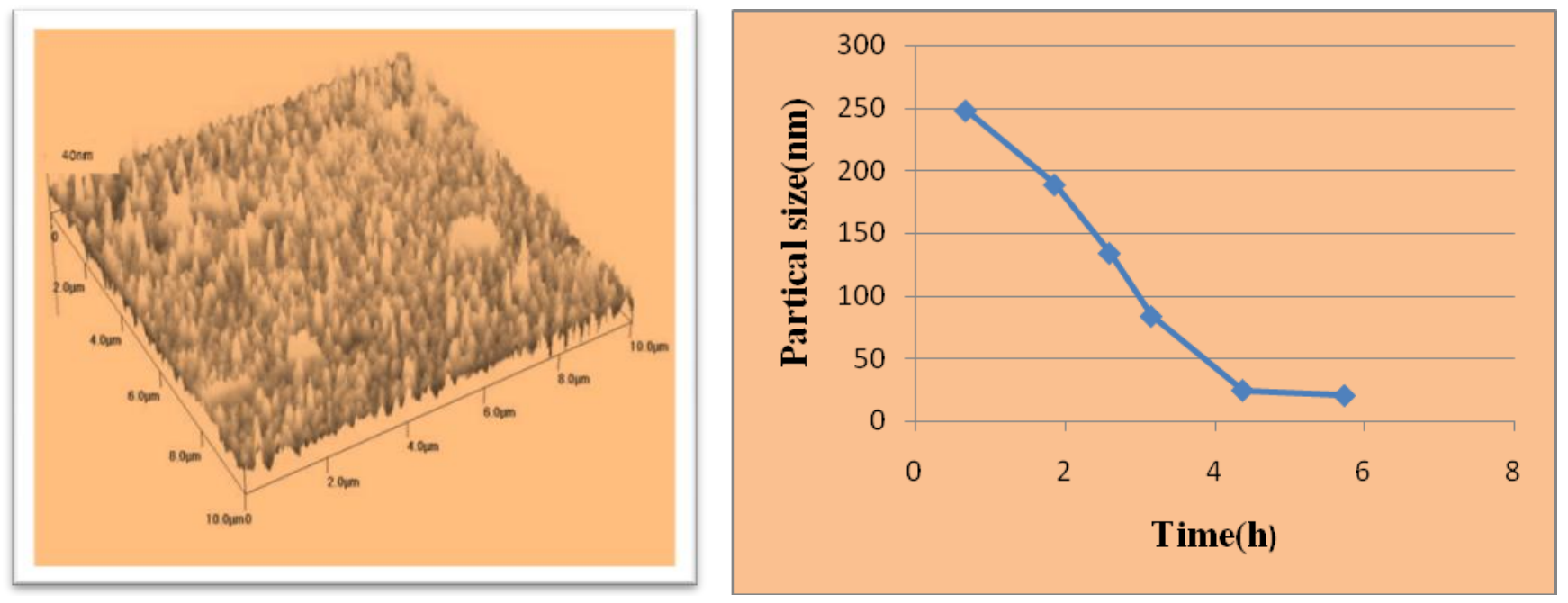

Figure 2: AFM image of silica show to a particle size less than $40-50 \mathrm{~nm}$.
Figure3: Show to a particle size\& Time mix 

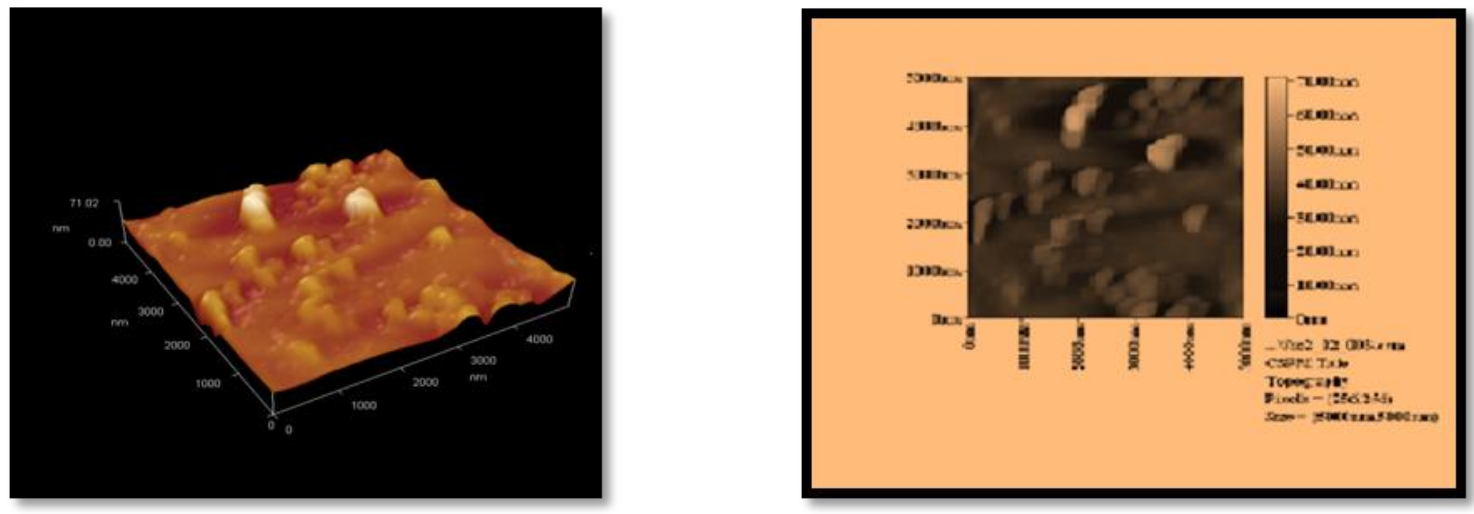

Figure 4: AFM image of silica show to grain size few to $70 \mathrm{~nm}$

Figure5: image AFM show to a particle size less than 40-50 nm:

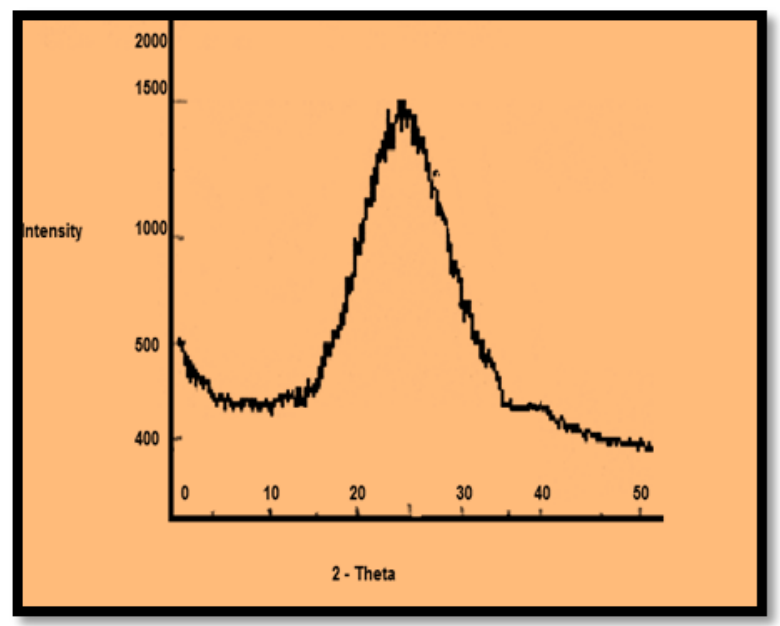

Figure 6: XRD-. X-ray diffraction of nano- $\mathrm{SiO}_{2}$ and amorphous silica

According to the results of x-ray diffraction (Fig.6), the obtained nano-SiO2 is a highly amorphous material with predominant crystallite size of 1.19-2.40 micrometer, after this to be perfect to burning during at $850 \mathrm{C}$ transmutation nano- crystal of silica.

\section{DISCUSSION}

Nanosilica can be successfully prepared by precipitation methods under controlled conditions. Nanosilica prepared by this method has a particle size less than $40-50 \mathrm{~nm}$ which is lower than that of the commercially available silica. Using appropriate dispersing agents in the reaction, the particle size can be controlled.SI as a possible substitute for condensed silica fume in concrete Pozzolanic materials are used to prevent or minimize cracking in concrete due to the expansive gel formed by the alkali-silica reaction. Silica fume and SI have been classified as "Highly active pozzolans[13]. on the synthesis, properties and applications of silica fume particularly for its pozzolanic activity[14]. 


\section{REFERENCES}

1-S.K, Dolui.J. Sol-Gel science Technology .20,107, (2004) .

2-C.N.R.Rao\&A.GovindRag. Nanotube and Nanwires, the Royal Society of Chemistry .London (2005).

3- L, Mathew.S,K,Narayanakuty."Synthesis and Characterisation of Nanosilica"International Conference on Avances in polymer in Technology,India PP.279,26-27,(2010).

4-S.Kourounis,S.Tsivilis,P.E.Tsakiridis" Cement and Concrete Research "pp37,815,(2007).

5-S.Kumer,R.A.Bandopadhyay,T.C.Alex. "Cement and Concrete Composites"30,679,(2008).

6-J.H.Johnston,A.J.Mcfarlane"Current Apple .Physics "4,411,(2004).

7-K.N.Pham,D.Fullston,K.Sagoe -Crentsil, J. Colloid Interf.Science;315,123,(2007).

8-B.J.Hwang,S.W.Park,D.W.Park"Chemical Engineering",26,775,(2009).

9- Joel, M.S." Chemically modified surfaces in catalysis and electrocatalysis". ACS Symposium Series. Washington. D.C.: American Chemical Society 192, 1, (1982)..

10- Farook, A. \& Chua, J.H. The adsorption of palmytic acid on rice husk ash chemically modified with $\mathrm{Al}(\mathrm{III})$ ion using the sol-gel technique. J. Colloid and Interface Sci., 280, 55-61, (2004).

11- Farook, A. \& Saleh, M.I." The removal of FFA from CPO and adsorption of palmytic acid on rice husk ash " In M.R. Nordin, K.Y. Liew, \& A.A. Zainal (Eds.) Surface science and heterogeneous catalysis. Pulau Pinang: Penerbit niversiti Sains Malaysia, 99. (1993).

12- Kamath, S.R. \& Proctor," ASilica gel from rice hull ash: Preparation and characterization". Cereal Chem., 75, 484487. (1998).

13-V. S. Ramachandran, “ Concrete Admixtures Handbook” 2nd ed. (Noyes Publications, USA,( 1995).

14- H. Asgeirsson andG. Gudmundsson," Comical. Concrerte". Res. 9,2,249,(1979).

15- P." Scherrer, Göttinger Nachrichten Gesell"., Vol. 2, 1918, p 98.-(1918).

16- Patterson, A. (1939). "The Scherrer Formula for X-Ray Particle Size Determination". Phys. Rev. 56 (10): 978-982. Bibcode 1939PhRv,56,978P(1938). 